\title{
Spontaneous migraine attack causes alterations in default mode network connectivity: a resting-state $\mathrm{fMRI}$ case report
}

\author{
Andrea Edit Edes ${ }^{1,2,3^{*}+}$, Lajos Rudolf Kozak ${ }^{1,4+}$, Mate Magyar ${ }^{1,5}$, Terezia Zsombok ${ }^{1,2,5}$, Gyongyi Kokonyei ${ }^{1,2,6}$, \\ Gyorgy Bagdy $2,3,7$ and Gabriella Juhasz $z^{1,2,3,8}$
}

\begin{abstract}
Background: Although migraine is one of the most investigated neurologic disorders, we do not have a perfect neuroimaging biomarker for its pathophysiology. One option to improve our knowledge is to study resting-state functional connectivity in and out of headache pain. However, our understanding of the functional connectivity changes during spontaneous migraine attack is partial and incomplete.

Case presentation: Using resting-state functional magnetic resonance imaging we assessed a 24-year old woman affected by migraine without aura at two different times: during a spontaneous migraine attack and in interictal phase. Seed-to-voxel whole brain analysis was carried out using the posterior cingulate cortex as a seed, representing the default mode network (DMN). Our results showed decreased intrinsic connectivity within core regions of the DMN with an exception of a subsystem including the dorsal medial and superior frontal gyri, and the mid-temporal gyrus which is responsible for pain interpretation and control. In addition, increased connectivity between the DMN and pain and specific migraine-related areas, such as the pons and hypothalamus, developed during the spontaneous migraine attack.
\end{abstract}

Conclusion: Our preliminary results provide further support for the hypothesis that alterations of the DMN functional connectivity during migraine headache may lead to maladaptive top-down modulation of migraine pain-related areas which might be a specific biomarker for migraine.

Keywords: Neuroimaging, Functional connectivity, Migraine, Migraine attack, Headache pain, Pain processing, Case report

\section{Background}

Pain connectome investigations in migraine, using functional magnetic resonance (fMRI) studies during rest and pain suggest that the number of attacks depend on resting-state functional connectivity between the default mode network (DMN), the salience network (SN) and the periaqueductal gray $[1,2]$. However, most of these alterations are not migraine-specific and similar biases in

\footnotetext{
*Correspondence: edes.andrea@pharma.semmelweis-univ.hu ${ }^{\dagger}$ Andrea Edit Edes and Lajos Rudolf Kozak contributed equally to this work

${ }^{3}$ Department of Pharmacodynamics, Faculty of Pharmacy, Semmelweis University, Nagyvárad Square 4, Budapest H-1089, Hungary

Full list of author information is available at the end of the article
}

functional connectivity can be observed in other chronic pain disorders [3]. In addition, much less is known about the pain connectome signature of acute migraine attacks. Only one study has investigated the effect of pharmacologically induced migraine attack on resting-state functional connectivity, reporting widespread alterations in DMN connectivity with different brain areas [4]. To our knowledge there are no data about spontaneous migraine pain connectome available in the scientific literature so far. In our present work we analysed resting-state fMRI data of a patient with migraine without aura who participated in two fMRI scans and suffered a migraine attack on the second occasion. 


\section{Case presentation}

Here we report a case of a 24-year old female migraine without aura patient who participated in two restingstate fMRI sessions (31 days apart) in our study. The diagnosis of migraine was made by a headache specialist according to the International Headache Society criteria [5]. The headache attacks occurred approximately 4 times per month since age 14 . They were severe in intensity (7-8 on a scale of 1-10) and had a throbbing quality with nausea, vomiting and photophobia. A typical episode lasted 4-72 h. Her migraine was not related to menstrual cycle. The patient had no other medical conditions and had not taken any daily medications except a contraceptive (gestodene, ethinylestradiol). Both the interictal and ictal scans were carried out on pill-taking days. Migraine attacks were treated with diclofenac and domperidone for nausea.

During the 3 days preceding the fMRI scans she was pain- and medication-free both times. At the second occasion she suffered an unexpected migraine attack during the examination that she subsequently described as a bilateral, throbbing pain (7/10) aggravated by head motion and associated with photophobia and nausea, and after the scan she vomited. In the beginning of the ictal image recording the pain was moderate (3/10) and pulsating with mild photophobia.

Functional MRI data acquisition was performed in the MR Research Center (Semmelweis University, Budapest, Hungary) with a $3 \mathrm{~T}$ Achieva MRI scanner (Philips Medical Systems, Best, The Netherlands) using a BOLDsensitive $\mathrm{T} 2 \%$-weighted echo-planar imaging sequence $\left(\mathrm{TR}=2.500 \mathrm{~ms}, \mathrm{TE}=30 \mathrm{~ms}, \mathrm{FOV}=240 \times 240 \mathrm{~mm}^{2}\right)$ with $3 \times 3 \mathrm{~mm}$ in-plane resolution and contiguous $3-\mathrm{mm}$ slices providing whole brain coverage. A series of anatomical images were also acquired during the first imaging session using T1-weighted 3D TFE sequence with $1 \times 1 \times 1 \mathrm{~mm}$ resolution. To determine the significant alterations in the functional connectivity, a period of 4 min from both sessions was selected, while the patient was in rest and with eyes opened. Imaging data were analysed using statistical parametrical mapping 12 (SPM 12, Friston, The Welcome Department of Cognitive Neurology, London, UK) and the CONN toolbox for MAT$\mathrm{LAB}$, using seed-to-voxel whole brain analysis, with false discovery rate (FDR) correction at $\mathrm{p} \leq 0.05$ significance level and a minimum cluster size limit of 50 voxels. The DMN seed region was chosen according to the work of Amin and colleagues [4] [posterior cingulate cortex, Montreal Neurological Institute (MNI) coordinates -2 $-6036, \mathrm{R}=10 \mathrm{~mm}$ ) to make our results comparable to the reported connectivity changes obtained during PACAP38-induced migraine attacks.
The study was approved by the Scientific and Research Ethics Committee of the Medical Research Council, Budapest, Hungary (23609-1/2011-EKU) and was carried out according to the Declaration of Helsinki. The subject gave written informed consent.

\section{Results}

During spontaneous migraine attack the DMN showed increased functional connectivity with brain areas related to pain, e.g. thalamus, insula and left postcentral gyrus, and decreased connectivity within the DMN (e.g. precuneus, ventral medial prefrontal cortex, angular gyrus). However, a subsystem of the DMN containing the dorsal medial frontal cortex (dMFC), the superior frontal gyrus (SFG) and the mid temporal gyrus (MidTG) showed increased FC. In addition, increased functional connectivity appeared with the pons and the hypothalamus (Table 1; Fig. 1).

\section{Conclusions}

Our results demonstrated that during spontaneous migraine attack the intrinsically salient migraine pain decreased functional connectivity within core regions of the DMN, and in parallel connectivity increased between the DMN and SN as a marker of attentional shift towards pain, and also increased between the DMN and regions of pain matrix [2]. These findings support the idea of dynamically changing functional connectivity during migraine cycle, similarly to an earlier longitudinal resting state fMRI study [6].

However, some functional connectivity changes during migraine pain may contribute to longstanding alterations in brain function and thus promote the recurrence of migraine attacks. For example, a subsystem of the DMN, namely the dMFC, SFG and MidTG showed increased functional connectivity with the DMN seed represented by $\mathrm{PCC}$, both in our spontaneous migraine case and during pharmacologically induced migraine attacks [4], which plays an important role in pain interpretation and control [7], and showed a significant difference between migraine without aura patients and controls during interictal phase [8]. In addition, the dMFC which is not only part of the DMN but belongs to the pain connectome, has an important role in pain modulation through shifting attention towards and away from the unpleasant sense [9] and has increased functional connectivity with other DMN regions in chronic pain patients $[10,11]$. This altered FC has been found to be positively correlated with pain rumination which is an indicator of unfavourable clinical outcome in chronic pain patients [10].

A previous study reported enhanced functional coupling between the hypothalamus and the middle pons 


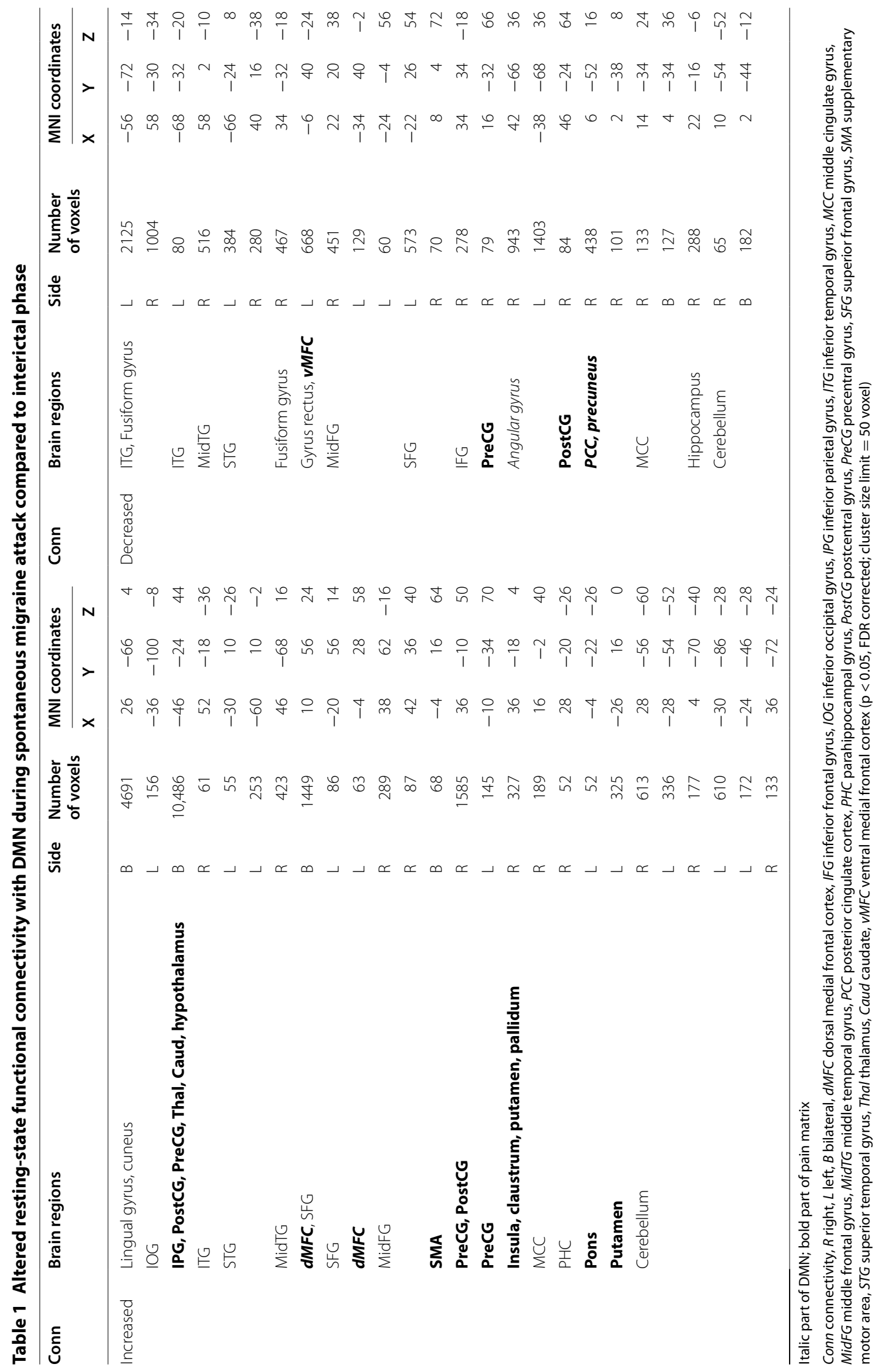




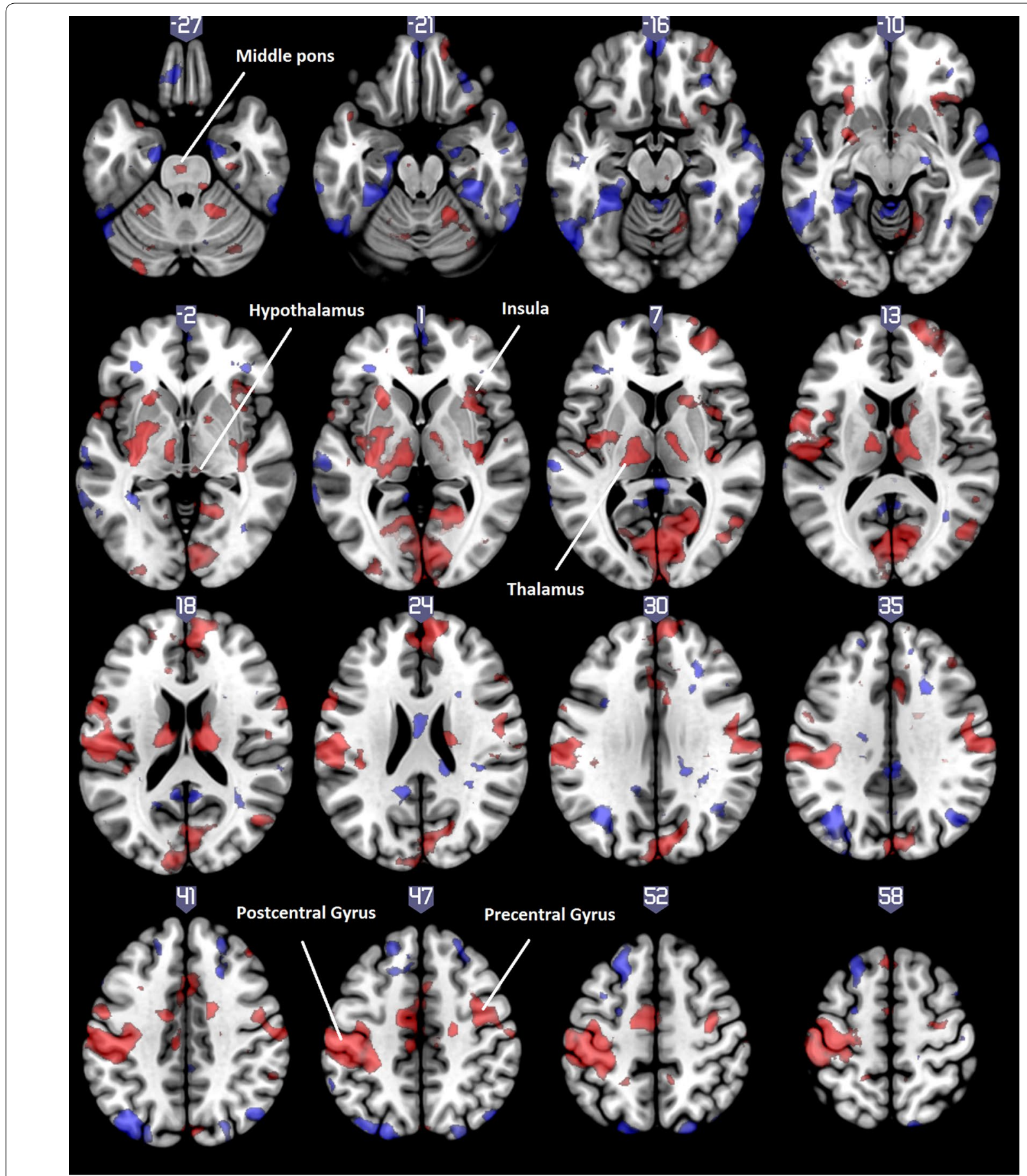

Fig. 1 Altered resting-state functional connectivity with DMN during spontaneous migraine attack compared to interictal phase, axial view, painrelated regions written out. Red: ictal > interictal, Blue: interictal > ictal. ( $p<0.05$, FDR corrected; no cluster size limit)

during migraine attack in a patient in response to painful stimuli [12]. In our case, the DMN seed functional connectivity increased with a special region in the pons that was previously identified as migraine generator [13], and with the hypothalamus that is in line with the recent hypothesis which suggested that migraine attacks might be initiated by functional connectivity changes between the hypothalamo-brainstem areas [12].

Despite that the present work is about a single case, and both our and previous studies investigated only 
female migraine patients $[4,12]$, these preliminary results further emphasize that alterations of the DMN functional connectivity during migraine attacks may lead to neuroplastic changes in the frontal cortical pain modulating network, which can lead to maladaptive top-down control of the hypothalamo-brainstem areas. Thus resting-state functional connectivity changes in migraineurs might be neuroimaging biomarkers for migraine pathophysiology. In the future, large prospective studies are required to investigate the resting state functional connectivity changes during both spontaneous and pharmacologically induced migraine attacks and to compare them to normal fluctuations in healthy control groups, to verify this hypothesis.

\section{Authors' contributions}

GJ designed the original study. AEE, GK and GJ collected the data. AEE and LRK analysed the data. AEE, TZ and MM drafted the paper. All authors critically reviewed the paper. All authors read and approved the final manuscript.

\section{Author details}

${ }^{1}$ MTA-SE-NAP B Genetic Brain Imaging Migraine Research Group, Hungarian Academy of Sciences, Semmelweis University, Nagyvárad Square 4, Budapest H-1089, Hungary. ${ }^{2}$ MTA-SE Neuropsychopharmacology and Neurochemistry Research Group, Hungarian Academy of Sciences, Semmelweis University, Nagyvárad Square 4, Budapest H-1089, Hungary. ${ }^{3}$ Department of Pharmacodynamics, Faculty of Pharmacy, Semmelweis University, Nagyvárad Square 4, Budapest H-1089, Hungary. ${ }^{4}$ MR Research Center, Semmelweis University, Balassa Street 6, Budapest H-1083, Hungary. ${ }^{5}$ Department of Neurology, Faculty of Medicine, Semmelweis University, Balassa Street 6, Budapest H-1083, Hungary. ${ }^{6}$ Institute of Psychology, Eotvos Lorand University, Izabella Street 46, Budapest H-1064, Hungary. ${ }^{7}$ NAP-A-SE Research Group, Hungarian Academy of Sciences, Semmelweis University, Nagyvárad Square 4, Budapest H-1089, Hungary. ${ }^{8}$ Neuroscience and Psychiatry Unit, Manchester Academic Health Sciences Centre, The University of Manchester, Stopford Building, Oxford Road, M13 9PT, Manchester, UK.

\section{Acknowledgements}

The study was supported by the MTA-SE-NAP B Genetic Brain Imaging Migraine Research Group, Hungarian Academy of Sciences, Semmelweis University (Grant No. KTIA_NAP_13-2-2015-0001), and the Hungarian Academy of Sciences (MTA-SE Neuropsychopharmacology and Neurochemistry Research Group). L. R. K. was supported by the Bolyai Research Fellowship Program of the Hungarian Academy of Sciences. The funders had no role in the design and conduct of the study; collection, management, analysis, and interpretation of the data; preparation, review, or approval of the manuscript; and decision to submit the manuscript for publication.

\section{Competing interests}

The authors declare that they have no competing interests.

\section{Availability of data and materials}

The datasets during and/or analysed during the current study available from the corresponding author on reasonable request.

\section{Consent to publish}

Written informed consent was obtained from the patient for publication of this Case Report and any accompanying images.

\section{Ethics approval and consent to participate}

The study was approved by the Scientific and Research Ethics Committee of the Medical Research Council, Budapest, Hungary (23609-1/2011-EKU). The subject gave written informed consent.

\section{Publisher's Note}

Springer Nature remains neutral with regard to jurisdictional claims in published maps and institutional affiliations.

Received: 12 September 2016 Accepted: 6 April 2017

Published online: 26 April 2017

\section{References}

1. Mainero C, Boshyan J, Hadjikhani N. Altered functional magnetic resonance imaging resting-state connectivity in periaqueductal gray networks in migraine. Ann Neurol. 2011;70(5):838-45.

2. Kucyi A, Davis KD. The dynamic pain connectome. Trends Neurosci. 2015:38(2):86-95.

3. Baliki MN, Mansour AR, Baria AT, Apkarian AV. Functional reorganization of the default mode network across chronic pain conditions. PLOS ONE. 2014;9(9):e106133.

4. Amin FM, Hougaard A, Magon S, Asghar MS, Ahmad NN, Rostrup E, et al. Change in brain network connectivity during PACAP38-induced migraine attacks: a resting-state functional MRI study. Neurology. 2016;86(2):180-7.

5. Bes A, Kunkel R, Lance JW, Nappi G, Pfaffenrath V, Rose FC, et al. The international classification of headache disorders, 3rd edition (beta version). Cephalalgia. 2013;33(9):629-808.

6. Zhao L, Liu J, Yan X, Dun W, Yang J, Huang L, et al. Abnormal brain activity changes in patients with migraine: a short-term longitudinal study. J Clin Neurol. 2014;10(3):229-35.

7. Tracey I. Imaging pain. Brit J Anaesth. 2008;101(1):32-9.

8. Tessitore A, Russo A, Giordano A, Conte F, Corbo D, De Stefano M, et al. Disrupted default mode network connectivity in migraine without aura. J Headache Pain. 2013;14:89.

9. Kucyi A, Salomons TV, Davis KD. Mind wandering away from pain dynamically engages antinociceptive and default mode brain networks. Proc Natl Acad Sci USA. 2013;110(46):18692-7.

10. Kucyi A, Moayedi M, Weissman-Fogel I, Goldberg MB, Freeman BV, Tenenbaum HC, et al. Enhanced medial prefrontal-default mode network functional connectivity in chronic pain and its association with pain rumination. J Neurosci. 2014;34(11):3969-75.

11. Gracely RH, Geisser ME, Giesecke T, Grant MA, Petzke F, Williams DA, et al. Pain catastrophizing and neural responses to pain among persons with fibromyalgia. Brain. 2004;127(Pt 4):835-43.

12. Schulte LH, May A. The migraine generator revisited: continuous scanning of the migraine cycle over 30 days and three spontaneous attacks. Brain. 2016;139:1987-93.

13. Weiller C, May A, Limmroth V, Juptner M, Kaube H, Schayck RV, et al. Brain stem activation in spontaneous human migraine attacks. Nat Med. 1995; 1(7):658-60 